\title{
INHALT
}

ABKÜRZUNGSVERZEICHNIS ........... X XV

VORWORT/Karl Kaiser und Hanns W. Maull . . . . . . . . XIX

EINE NEUE INTERNATIONALE UMWELT: VERÄNDERUNGSTENDENZEN IM INTERNATIONALEN SYSTEM

INTERNATIONALE POLITIK ZWISCHEN INTEGRATION UND ZERFALL/Hanns W. Maull . . . . . . . . . . . . . 1

TeChNOLOgISChE DYNAMIK UND SOZIALER WANDEL $\ldots \ldots$

AUSPRÄGUNGEN DER DIALEKTIK VON INTEGRATION UND ZER-

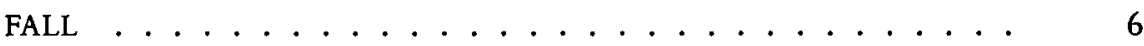

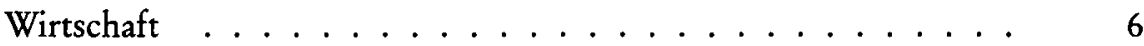

Gesellschaft und Kultur . . . . . . . . . . . . . . 9

Internationale Ordnung, Stabilität und Sicherheit . . . . . . . 12

Die DiALEKTIK VON INTEGRATION UND ABgRENZUNG ALS HERAUSFORDERUNG DER POLITIK $\ldots \ldots \ldots \ldots \ldots$

WELTWIRTSCHAFT IM UMBRUCH/Reinhard Rode . . . . . 23

STRUKTURWANDEL IN DER WELTWIRTSCHAFT . . . . . . . . 24

Wachstum und Größe . . . . . . . . . . . . . . 25

Handel ........................... 26

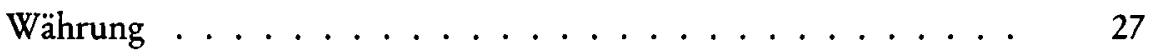

Investitionen und Transnationale Konzerne . . . . . . . . 28

Finanzen ................... 30

High-Tech ....................... 31

HERAUSFORDERUNGEN FÜR DIE WIRTSCHAFTSPOLITIK . . . . 32

Internationale Institutionen . . . . . . . . . . . . . 32 
Regionalisierung ...................... 34

Wettbewerb von Gesellschaftsmodellen . . . . . . . . . 35

Arbeitsmärkte . . . . . . . . . . . . . . 38

ASYMMETRIEN UND DIE FOLGEN $\ldots \ldots \ldots$

ENTWICKLUNG UND UNTERENTWICKLUNG. TRENDS UND HERAUSFORDERUNGEN/Gabriele Brenke . . . . . 43

EINLEITUNG $\ldots \ldots \ldots \ldots \ldots \ldots \ldots$

BILANZ DER WICHTIGSTEN ENTWICKLUNGSTRENDS . . . . . . . 44

Asien zwischen Wirtschaftswunder, nachholender Entwicklung und großer Armut . . . . . . . . . . . . . . 45

Transformation in Lateinamerika . . . . . . . . . . . 47

Afrika - der verlorene Kontinent? . . . . . . . . . . . . 49

URSACHEN FÜR ENTWICKLUNG UND UNTERENTWICKLUNG . . 51

Bevölkerungswachstum und Armut . . . . . . . . . . . 51

Bürgerkriege und Zerfallsprozesse . . . . . . . . . . . 53

Schuldenkrise ...................... 54

Demokratisierung und Good Governance: eine Voraussetzung für Entwicklung? . . . . . . . . . . . . . 55

BEDROHUNGS- UND KONFLIKTPOTENTIALE . . . . . . . . 56

FOLGERUNGEN FÜR DIE DEUTSCHE POLITIK $\ldots \ldots \ldots$

DIE REVOLTE GEGEN DEN WESTEN IN DER NEUEN INTERNATIONALEN UMWELT: DAS BEISPIEL DER ISLAMISCHEN ZIVILISATION/ Bassam Tibi

DAS PHÄNOMEN DES RELIGIÖSEN FUNDAMENTALISMUS IN DER NEUEN INTERNATIONALEN UMWELT: DIE ENTWESTLICHUNG DER WELTPOLITIK $\ldots \ldots \ldots \ldots \ldots$

DIE AUSSENPOLITISCHE ORIENTIERUNG DES ISLAMISCHEN FUNDAMENTALISMUS: DER RELIGIÖSE RADIKALISMUS ALS EINE ANTIWESTLICHE STRATEGIE $\ldots \ldots \ldots \ldots \ldots$ 
DER WELTPOLITISCHE RAHMEN DES ISLAMISCHEN FUNDAMENTALISMUS UND DIE ZWEITEILUNG DER WELT $\ldots \ldots \ldots$

DAS SCHEITERN DES WESTLICH GEPRÄGTEN ENTWICKLUNGSMODELLS ALS HINTERGRUND RELIGIÖS-POLITISCHER OPTIONEN FÜR EINE ENTSÄKULARISIERUNG VON STAATS- UND WELTORDNUNG

DeR Fundamentalismus als PROgRamm gegen die VerpflaNZUNG DES WESTLICHEN MODELLS VOM SÄKULAREN NATIONALSTAAT IN DIE WELT DES ISLAM . . . . . . . . . . . . . .

KANN DER FUNDAMENTALISMUS DAS ISLAMISCHE »UMMA«-IDEAL IN EINE ANTIWESTLICHE WELTPOLITISCHE GRÖSSE VERWANDELN?

SCHLUSSFOLGERUNGEN UND IMPLIKATIONEN FÜR DIE AUSSEN-

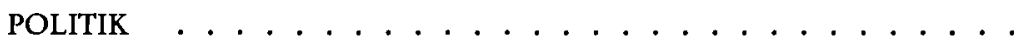

\section{REGIONALE HERAUSFORDERUNGEN}

OSTASIENS HERAUSFORDERUNG/Rüdiger Machetzki . . . 81

OSTASIEN WIRD $»$ ENTDECKT $\ll \ldots \ldots \ldots \ldots \ldots$

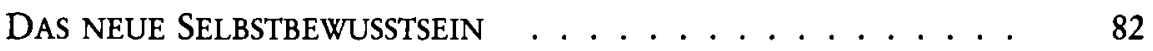

OSTASIATISCHE LEITERFAHRUNGEN . . . . . . . . . 83

ASIATISCHE KONFLIKTDIMENSIONEN UND DER WESTEN . . . 86

VON DER DiSKUSSION UM KULTUR UND POLITIK . . . . . . . . 90

VOM EINFLUSS DER TRADITION - ETHIK UND »PRAGMATISMUS $\lll 91$

ELITEBILDUNG UND LERNTRADITION . . . . . . . . . . . . . . 94

ZUM ZUKUNFTSOPTIMISMUS - OSTASIEN IST STARK . . . . . . . 98

JAPAN - NACHLASSEN DER VITALITÄT? . . . . . . . . . . . . 99

LERNFÄHIGKEIT ALS ENTWICKLUNGSKONZEPT . . . . . . . 102 
DIE NEUEN DEMOKRATIEN IN OSTMITTELEUROPA UND DIE EUROPÄISCHE UNION/Roland Freudenstein . . . . . 103

EINFÜHRUNG $\ldots \ldots \ldots \ldots \ldots \ldots$

DIE MITTEL- UND OSTEUROPÄISCHEN STAATEN FÜNF JAHRE NACH DER EUROPÄISCHEN WENDE . . . . . . . . . . . . . . . 104 Stand der Transformation . . . . . . . . . . . . . . . 104

Strategien gegenüber der Europäischen Union . . . . . . . . 105

DIE POLITIK DER EUROPÄISCHEN UNION SEIT $1989 \ldots \ldots$ Finanztransfer . . . . . . . . . . . . . 107

Technische Hilfe . . . . . . . . . . . . . . . . 108 Europa-Abkommen . . . . . . . . . . . . 108 Marktöffnung . . . . . . . . . . . . . . 109

Politischer Dialog . . . . . . . . . . . . . . . 109

Strukturierte institutionelle Beziehungen . . . . . . . . 110

»Assoziierte Partnerschaft « mit der Westeuropäischen Union . . . 110

DIE HERAUSFORDERUNGEN MITTE DER NEUNZIGER JAHRE . . 111

Eine Strategie zur Vorbereitung der Mitgliedschaft . . . . . . . 111

Die "erweiterte MOE-Gruppe und das Problem der Beitrittssequenz . . . . . . . . . . . . . . . . . . 112

Konsequenzen für die interne Struktur: Aufgaben für die Regierungskonferenz 1996 . . . . . . . . . . . . . . . . . 113

Die Reform des sicherheitspolitischen Institutionengeflechts . . . 115

Divergierende Prioritäten zwischen den Mitgliedstaaten . . . . 116 Zwei Blöcke in Europa? . . . . . . . . . . . . . . . . . 116

AUSBLICK: KONSEQUENZEN FÜR DIE POLITIK DEUTSCHLANDS 117

RUSSLAND IN EUROPA/Alexander Rahr $\ldots \ldots \ldots \ldots$

UNTERSCHIEDLICHE ANSICHTEN ÜBER DIE ZUKUNFT GESAMT-

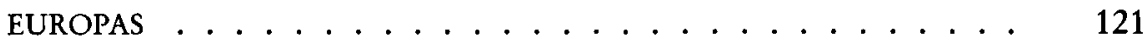

ZWISCHEN REFORM UND RESTAURATION . . . . . . . . . . 122

Westöffnung oder Sonderweg . . . . . . . . . . . . . . 122

Innere Stabilität auf Kosten der Rechtsstaatlichkeit . . . . . . . . 124

Unvollendete Wirtschaftsreformen . . . . . . . . . . . . 126

Außenpolitische Neuorientierung . . . . . . . . . . . . . . 128 
Die Politik des »Nahen Auslands« . . . . . . . . . . . . 130

CHANCEN UND RISIKEN . . . . . . . . . . . . . . . . . 131

VIER SZENARIEN FÜR MÖGLICHE ENTWICKLUNGEN . . . . . . 132

Das optimistische Szenarium . . . . . . . . . . . . . . 132

Das UdSSR-Reanimierungs-Szenarium . . . . . . . . . . . . 133

Das chinesische Szenarium . . . . . . . . . . . . 133

Das Mafia- oder Zerfallsszenarium . . . . . . . . . . . 133

WEGE ZUR PARTNERSCHAFT . . . . . . . . . . . . . . . 134

HANDLUNGSSPIELRAUM DEUTSCHER OSTPOLITIK $\ldots \ldots \ldots$

NATIONALITÄTENKONFLIKTE AUF DEM BALKAN/

Klaus Becher . . . . . . . . . . . . . . . 137

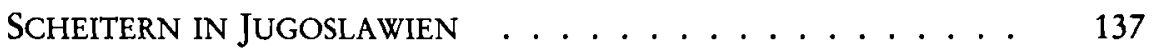

FAKTOREN INNERER DYNAMIK $\ldots \ldots \ldots \ldots \ldots$

NATIONALITÄTENKONFLIKT ALS MACHTINSTRUMENT . . . . . 143

RISIKEN UND GEFAHREN . . . . . . . . . . . . . . . 145

AUFGABEN DEUTSCHER UND EUROPÄISCHER POLITIK $\ldots \ldots$

FREMDE NACHBARN: DER NAHE UND MITTLERE OSTEN/ Gudrun Krämer . . . . . . . . . . . . . . . . . 157

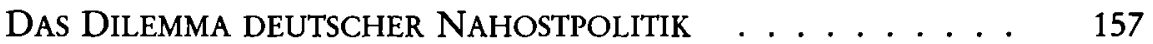

DEUTSCHE INTERESSEN IM NAHEN UND MITTLEREN OSTEN . . 158

PROBLEMFELDER STATT $»$ KRISENBOGEN $« \ldots \ldots \ldots$

Die Konfliktregion um Israel . . . . . . . . . . . . . . . . 160

Die Golfregion . . . . . . . . . . . . . . . . . . . . 162

Zentralasien und Kaukasus . . . . . . . . . . . . . 163

Die Türkei . . . . . . . . . . . . . . . . . . . . 163

Der Maghreb . . . . . . . . . . . . . . . . . 164

KONFLIKTBEWÄLTIGUNG IM NAHEN UND MITTLEREN OSTEN . 164

DER PRIMAT DER INNENPOLITIK $\ldots \ldots \ldots \ldots$ 
LIBERALISIERUNG $\ldots \ldots \ldots \ldots \ldots \ldots$

DIE INTEGRATION DER ISLAMISTEN . . . . . . . . . . 170

SCHLUSS: HERAUSFORDERUNGEN IM NAHEN UND MITTLEREN

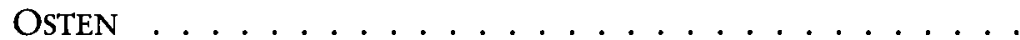

\section{KONFLIKTFELDER}

MIGRATION: HERAUSFORDERUNG DEUTSCHER UND EUROPÄISCHER POLITIK/Steffen Angenendt . . . . . . . . 175

BESTIMMUNGSFAKTOREN VON WANDERUNGSBEWEGUNGEN $\ldots .176$

Politische Wanderungsfaktoren . . . . . . . . . . . . 176

Soziokulturelle Wanderungsfaktoren . . . . . . . . . . 177

Ökonomische Wanderungsfaktoren . . . . . . . . . . . . 178

Demographische Wanderungsfaktoren . . . . . . . . . 179

Ökologische Wanderungsfaktoren . . . . . . . . . . 180

ZUWANDERUNGSPOTENTIALE FÜR DEUTSCHLAND UND EUROPA 181

Zuwanderungspotentiale in Ost- und Südosteuropa . . . . . . . 182

Die ehemalige Sowjetunion . . . . . . . . . . . . . . . . 184

Nordafrika . . . . . . . . . . . . . . . . 186

HERAUSFORDERUNGEN UND CHANCEN . . . . . . . . . . . 187

Arbeitsmarkt, demographische Entwicklung und Wirtschaftswachstum

Sozialstaat . . . . . . . . . . . . . . . . 189

Innere Sicherheit . . . . . . . . . . . . . . . . 190

Äußere Sicherheit . . . . . . . . . . . . . . . . . . 194

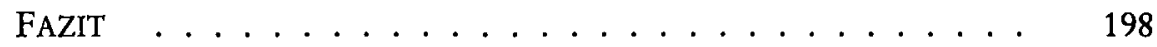

RÜSTUNGS- UND ZERSTÖRUNGSPOTENTIALE ALS HERAUSFORDERUNG DER INTERNATIONALEN POLITIK/ Harald Müller . . . . . . . . . . . . . . . . . 201

DAS PROLIFERATIONSPROBLEM NACH DEM ENDE DES OST-WESTKONFLIKTS: PLUS ÇA CHANGE ... . . . . . . . . . 
DENNOCH: GRUND ZUR UNRUHE . . . . . . . . . . . . . . 202

PROLIFERATION DURCH STAATEN: BILANZ UND AUSBliCK . . . 204

RÜSTUNGSTRENDS UND WELTPOLITIK: EINE EINSCHÄTZUNG DER FOLGEN . . . . . . . . . . . . . . . 206

RISIKEN UND BEDROHUNGEN . . . . . . . . . . . . . 208

RISIKEN UND BEDROHUNGEN AUS DER SICHT DER BUNDESREPUBLIK ....................

REGIME UNTER STRESS: INTERNATIONALE BEMÜHUNGEN ZUR EINDÄMMUNG DER PROLIFERATION . . . . . . . . . . . . . . 214

Das nukleare Nichtverbreitungs-Regime . . . . . . . . . . 214

Die Chemiewaffenkonvention ............. 216

Die Konvention über biologische Waffen . . . . . . . . . 217

Das Raketentechnologie-Kontrollregime . . . . . . . . . 218

Regelungen bei den konventionellen Waffen . . . . . . . 219

GLOBALE UND REGIONALE REgELUNGEN . . . . . . . . . . 220

PROLIFERATION IST KEIN NORD-SÜD-KONFLIKT $\ldots . \ldots . . . .222$

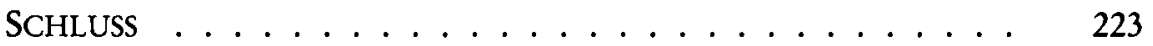

TRANSNATIONALE GEFÄHRDUNGEN DER INTERNATIONALEN SICHERHEIT/Hans-Georg Wieck . . . . . . . 225

EINLEITUNG $\ldots \ldots \ldots \ldots \ldots \ldots \ldots \ldots$

INTERNATIONALER UND NATIONALER TERRORISMUS . . . . . . 225

GRENZÜBERSCHREITENDE ORGANISIERTE KRIMINALITÄT . . . . 229

RAUSCHGIFTHANDEL UND GELDWÄSCHE . . . . . . . . . 230

TRANSNATIONALE KRIMINALITÄT AUS MITTELOSTEUROPA UND AUS DEM GEBIET DER FRÜHEREN SOWJETUNION . . . . . . . 233

AUFGABEN DEUTSCHER POLITIK UND DIE ROLLE DER DEUTSCHEN AUSSENPOLITIK ........................ 235 
UMWELTKRISEN UND INTERNATIONALE SICHERHEIT/

Hans Joachim Schellnhuber und Detlef F. Sprinz . . . . . . . 239

EINLEITUNG $\ldots \ldots \ldots \ldots \ldots \ldots$

NATÜRLICHE UMWELTKRISEN UND INTERNATIONALE SICHERHEIT 242

Der Globale Wandel . . . . . . . . . . . . . . 243

ANTHROPOGENE UMWELTKRISEN UND INTERNATIONALE SICHERHEIT . . . . . . . . . . . . . . . . . . . 245

Bedrohungen infolge hausgemachter Umweltzerstörung . . . . 245

Bedrohungen infolge nachbarschaftsbedingter Umweltprobleme . 249

Bedrohungen infolge fernverursachter Umweltdegradation . . . . 252

DER UMWELT-SICHERHEITS-KOMPLEX: EINE HERAUSFORDERUNG FÜR DIE DEUTSCHE AUSSENPOLITIK? . . . . . . . . . . . 256

AUSBLICK: INTERNATIONALE SICHERHEIT DURCH UMWELTGEFÄHRDUNG . . . . . . . . . . . . . . . . . . 259

PERSONENREGISTER . . . . . . . . . . . . . . 261

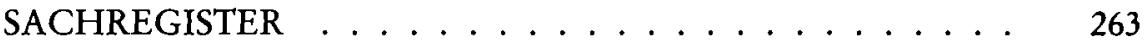

DIE AUTOREN . . . . . . . . . . . . . . . . . . 269 